\title{
Permethrin (PER) plus trimethoprim/sulfamethoxazole (TMP/SMX) was better than PER but not TMP/SMX for head lice infestation in children
}

Hipolito RB, Mallorca FG, Zuniga-Macaraig ZO, et al. Head lice infestation: single drug versus combination therapy with
one percent permethrin and trimethoprim/sulfamethoxazole. Pediatrics 2001 Mar; $107: \mathrm{E} 30$.

QUESTION: Is a combination of topical permethrin (PER) and oral trimethoprim/ sulfamethoxazole (TMP/SMX) better than either PER or TMP/SMX alone for treating head lice infestation (HLI)?

\section{Design}

Randomised (unclear allocation concealment*), blinded (unclear), ${ }^{*}$ controlled trial with 4 weeks follow up.

\section{Setting}

3 private paediatric and family practices in San Joaquin County, California, USA.

\section{Patients}

115 children who were $2-13$ years of age (70\% girls) and were diagnosed with HLI (clinical suspicion of lice or eggs). Children with hypersensitivity to TMP/SMX or $1 \%$ PER or with a history of parental non-compliance or neglect were excluded from the study. $97 \%$ of children completed follow up.

\section{Intervention}

In all treatment groups, parents were instructed to first wash their child's hair with regular shampoo and then rinse. 39 children were allocated to 1\% PER, for which parents were instructed to apply the 1\% PER creme rinse to the scalp and hair for 10 minutes, rinse, towel dry, and comb the hair thoroughly with the comb provided. The procedure could be repeated after 1 week if necessary. 36 children were allocated to oral TMP/ SMX at a dose of $10 \mathrm{mg} / \mathrm{kg}$ per day in 2 divided doses for 10 days. 40 children were allocated to the combined $1 \%$ PER and TMP/SMX treatment.

\section{Main outcome measure}

Cure of HLI (absence of adult lice and nymphal stage or eggs) at 2 and 4 weeks.

\section{Main results}

The combined treatment was better than PER alone (table) but not TMP/SMX alone for treating HLI at 2 weeks $(95.0 \% v 83.3 \%,\{\mathrm{p}=0.10\} \uparrow)$ and 4 weeks $(92.5 \% v$ $77.8 \%, \quad(\mathrm{p}=0.07\} \uparrow)$. PER alone was not better than TMP/SMX alone at 2 weeks $(79.5 \% v 83.3 \%,\{\mathrm{p}=0.67\} \dagger)$ and 4 weeks $(71.8 \% v 77.8 \%,\{\mathrm{p}=0.55\} \uparrow) .3$ children with an allergic rash appearing from TMP/SMX were excluded from the analysis. 3 children with mild scalp irritation from $1 \%$ PER use and 9 children with transient pruritis and 3 children with nausea and vomiting from TMP/SMX use were not excluded.

\section{Conclusions}

The combination of topical permethrin (PER) and oral trimethoprim/sulfamethoxazole (TMP/SMX) was a better second line treatment for head lice infestation than was PER alone but not TMP/SMX alone. TMP/SMX use was often associated with adverse reactions.

*See glossary.

tp Values calculated from data in article.
Source of funding: not stated.

For correspondence: Dr R B Hipolito, University of California at Davis, School of Medicine, Department of Pediatrics, Section of Neonatology, TB 193, Davis, CA 95616 USA.rbhipo@aol.com.

A modified version of this abstract also appears in

Evidence-Based

Nursing.

Combined 1\% permethrin creme rinse (PER) and trimethoprim/sulfamethoxazole v PER alone for cure of head lice infestation in children $\$$

\begin{tabular}{lllll} 
Outcomes & Combined & PER & RBI (95\% Cl) & NNT (CI) \\
Cure at 2 weeks & $95.0 \%$ & $79.5 \%$ & $19.5 \%(1.1$ to 48.4$)$ & 7 (4 to 117$)$ \\
\hline Cure at 4 weeks & $92.5 \%$ & $71.8 \%$ & $28.8 \%(5.3$ to 66.2$)$ & 5 (3 to 25) \\
\hline
\end{tabular}

$\neq$ Abbreviations defined in glossary; RBI, NNT, and $\mathrm{Cl}$ calculated from data in article.

\section{COMMENTARY}

Recurrences after the treatment of HLI are common. Most of these reinfestations are treatment failures, although some are new infestations, but evidence exists that some head lice are resistant to the drugs usually used in treating HLI. Because the turnover of different generations of lice is much less than in bacteria, the ability of lice to become drug resistant is rare.

Hipolito et al confirmed in their randomised controlled trial that a combination of $1 \%$ PER cream rinse with oral TMP/SMX was more effective than 1\% PER alone, with an NNT of 5 at 4 weeks. TMP/SMX is an antimicrobial drug with adverse effects caused mainly by the sulpha component. It is cheap, relatively safe, and widely used for acute otitis media and urinary tract infections. TMP/SMX works by killing the intestinal flora of the head lice via the blood they suck from the host. The effect of PER cream rinse is additive because it paralyses the respiratory muscles of the lice.

Some of the patients who participated in this study had recurrent HLI. The authors recommended that the use of TMP/SMX be reserved for patients with suspected drug resistance. Even though the primary outcome was confirmed by an office visit rather than by telephone in only half of the patients among the treatment groups, it is easy to recommend at least offering this combination treatment in patients who have reinfestation.

Matti Uhari, MD, MSc

University of Oulu Oulu, Finland 